\title{
The Pattern of Avian Intramuscular Nerve Branching Is Determined by the Innervating Motoneuron and Its Level of Polysialic Acid
}

\author{
Victor F. Rafuse and Lynn T. Landmesser \\ Department of Neurosciences, Case Western Reserve University, Cleveland, Ohio 44106
}

\begin{abstract}
Most skeletal muscles are composed of a heterogeneous population of fast and slow muscle fibers that are selectively innervated during development by fast and slow motoneurons, respectively. It is well recognized that, in both birds and mammals, fast and slow motoneurons have substantially different intramuscular branching patterns, a difference critical for proper motor function. However, the cellular mechanisms regulating these differences in motoneuron branching are unknown. In a previous study, we showed that the fast and slow pattern of intramuscular branching, in a chick muscle containing distinct fast and slow muscle regions, was remarkably similar to normal when formed by foreign motoneurons. Whether this was attributable to some property of the innervating "fast" or "slow" motoneurons or to some property of the developing fast-slow muscle fibers was not determined. To distinguish between these two possibilities, we performed
\end{abstract}

Skeletal muscles are composed of heterogeneous populations of muscle fibers that are categorized as slow or fast based on their relative speed of contraction (Henneman and Mendell, 1981). In adult, slow and fast muscle fibers are innervated by slow and fast motoneurons whose electrical properties are matched to the contractile properties of the fibers they innervate (Kernell, 1992). During contraction, the smaller, slow motoneurons are recruited before the larger, fast neurons (Henneman and Olson, 1965). Because slow motoneurons generate less force than fast neurons, the recruitment of motoneurons according to size ensures a smooth gradation of force during contraction. Slow motoneurons generate less force primarily because they branch and innervate fewer muscle fibers (Bodine et al., 1987; Totosy de Zepetnek et al., 1992). This branching difference is already established at birth (Brown et al., 1976). Although the orderly recruitment of force during muscle contraction is well recognized, and clinically important, the cellular mechanisms regulating intramuscular branching are poorly understood.

Addressing this problem in mammals is difficult because slow and fast muscle fibers are intermixed within muscles. Conse-

\footnotetext{
Received Sept. 16, 1999; revised Nov. 19, 1999; accepted Nov. 19, 1999.

This work was supported by National Institutes of Health Grants NS19640 and NS23678, as well as a McKnight Senior Investigator Award. We thank Shilpi Banerjee and Marianne Usiak for their helpful comments on this manuscript. In addition, we thank U. Rutishauser for supplying the endoneurominadase-N, D. Fambrough for the 5D2 monoclonal antibody, and F. E. Stockdale for the S58 antibody.

Correspondence should be addressed to Dr. Lynn Landmesser, Department of Neurosciences, Case Western Reserve University, 10900 Euclid Avenue, Cleveland, OH 44106-4975. E-mail: lt1@po.cwru.edu.

Dr. Rafuse's present address: Department of Surgery, Sir Charles Tupper Medical Building, Dalhousie University, Halifax, Nova Scotia, B3H 4H7, Canada.

Copyright (C) 2000 Society for Neuroscience $0270-6474 / 00 / 201056-10 \$ 15.00 / 0$
}

chick-quail hindlimb chimeras to force slow chick plantaris motoneurons to innervate a fast quail plantaris muscle. The pattern of intramuscular nerve branching in the fast plantaris of these chimeras closely resembled the slow branching pattern normally observed in chick slow plantaris muscles. Enzymatic removal of polysialic acid (PSA) from nerve and muscle during normal quail plantaris development dramatically changed the normal fast pattern to more closely resemble a slow pattern. In contrast, removal of PSA from chick plantaris motoneurons and muscle fibers had little effect on the pattern of nerve branching. Together, these results indicate that the pattern of intramuscular nerve branching is determined by the level of PSA on the innervating motoneurons.

Key words: muscle; myotubes; NCAM; fast/slow; synaptic transmission of fast muscle; slow muscle quently, slow and fast motoneurons are intermixed within the intramuscular nerves and, in the absence of distinguishing molecular markers, are impossible to identify during development. In contrast, slow and fast myofibers are grouped into discrete regions within avian muscles at early developmental stages (McLennan, 1983; Vogel and Landmesser, 1987). Avian slow and fast motoneurons also have characteristically different intramuscular branching patterns (Dahm and Landmesser, 1988). Earlier studies showed that the polysialic acid (PSA) moiety of neural cell adhesion molecule (NCAM) is more highly expressed on developing fast than on slow motoneurons. In ovo enzymatic removal of PSA from both developing nerve and muscle dramatically altered the normal fast pattern of innervation to resemble a slow pattern (Landmesser et al., 1990), showing that PSA is required for the fast pattern of intramuscular branching. However, because PSA was removed from both axons and muscle fibers, it was impossible to determine whether the altered intramuscular branching was caused by the absence of PSA from the innervating axons or the muscle fibers.

To determine whether the intramuscular nerve branching pattern is a property of motoneurons, or muscle fibers, we forced "slow" motoneurons to innervate a "fast" muscle. However, it is impossible to perform fast-slow nerve cross-union experiments at early developmental stages. Furthermore, we showed that, when embryonic chick motoneurons were given a choice of foreign muscles to innervate, they selectively innervated muscles, or muscle regions, containing fibers of the appropriate type (Rafuse et al., 1996). Nonetheless, it is possible to achieve fast-slow crossinnervation in chick-quail chimeras because developing motoneurons have a strong propensity to selectively innervate their homologous muscle, despite its muscle fiber composition (Tanaka 
and Landmesser, 1986a). Consequently, by using the fact that the chick plantaris muscle is slow whereas the quail is fast (Grim et al., 1989) and by performing chick-quail hindlimb chimeras, we forced slow chick plantaris motoneurons to innervate the fast quail plantaris muscle. Our results indicate that the pattern of intramuscular nerve branching is attributable to the level of PSA on the innervating motor axon.

Parts of this work have been published previously in abstract form (Rafuse and Landmesser, 1997).

\section{MATERIALS AND METHODS}

Embryonic surgery. Fertile eggs from white leghorn chicks and Japanese quails were incubated at $37^{\circ} \mathrm{C}$ in a circulated air humidified incubator. Chick-quail hindlimb chimeras were performed as described previously (Tanaka and Landmesser, 1986a). Briefly, under sterile conditions, a window was made in the eggs with a dental drill to expose stage (St.) 17-18 (Hamburger and Hamilton, 1951) embryos. The vitelline membrane and amnion were cut, and the embryo was stained with a $1 \%$ neutral red solution. Using a flame-sharpened tungsten wire $(0.077 \mathrm{~mm}$ wire; World Precision Instruments, Sarasota, FL), the right limb bud was removed from both the chick and quail embryos. The quail limb buds were then transferred to the chick egg and attached to the embryo by pressure, and the windows were sealed with a glass coverslip using liquid paraffin. The embryos were then placed back in the incubator, where they were allowed to develop until St. 35-37. As a consequence of the surgery, the chick embryos developed essentially normally, except that the right leg was derived entirely from the quail limb bud.

Muscle whole mounts. Plantaris muscle whole mounts were prepared as described previously (Rafuse et al., 1996). Briefly, all muscles in the shank, except the plantaris muscle, were removed. Using flamedsharpened tungsten needles, the connective tissue was carefully removed from the plantaris muscle, which was then washed in PBS, fixed for $2 \mathrm{~min}$ in $4^{\circ} \mathrm{C}$ acetone, washed in PBS, and then incubated overnight $\left(4^{\circ} \mathrm{C}\right)$ with a C2 monoclonal antibody that recognizes neurofilament protein, in $2 \%$ BSA containing $0.3 \%$ Triton X-100-PBS to allow penetration of the $\mathrm{C} 2$ antibody to the neurofilament binding sites. The muscle whole mounts were washed several times in PBS (five times for $10 \mathrm{~min}$ each), fixed for $20 \mathrm{~min}$ in $3.7 \%$ formaldehyde (room temperature), incubated for $1.5 \mathrm{hr}$ with a fluorescein-conjugated secondary antibody (IgG; Sigma, St. Louis, $\mathrm{MO}$ ), washed in PBS, and finally mounted between two coverslips in 50\% glycerin-PBS containing $0.03 \mathrm{mg} / \mathrm{ml} p$-phenylenediamine.

AChR labeling and isolation of single muscle fibers. Plantaris muscles from St. 43-44 embryos were dissected free from all the other shank muscles and incubated with tetramethylrhodamine-conjugated $\alpha$-bungarotoxin ( $\alpha$ BTX) (1:100 dilution; Molecular Probes, Eugene, OR) for $1 \mathrm{hr}$ at room temperature. The muscles were washed several times with PBS, and single fibers were teased apart after $1 \mathrm{hr} 2 \%$ collagenase (Sigma) digestion.

In ovo injection of endoneuraminidase N. At St. 29-30, holes were made in the chick and quail shells, and a coverslip was sealed in place with melted paraffin. One microliter of an endoneuraminidase $\mathrm{N}$ (Endo-N) solution containing 30,000 U/ml was injected into the right shank muscles at St. 29-30 using a broken-off micropipette. As shown in detail previously (Landmesser et al., 1990; Ono et al., 1994), this quantity of Endo-N injected into developing chick hindlimb or mouse brain effectively removes PSA from developing nerve and muscle fibers for several days in vivo.

Frozen-section immunohistochemistry. St. 35-37 chick or quail embryos were quickly removed from the egg, decapitated, eviscerated, and placed in Tyrode's solution. The shank was removed, mounted in O.C.T. (Miles, Elkhart, IN) and quickly frozen in isopentane that was cooled with dry ice. The frozen blocks were stored at $-70^{\circ} \mathrm{C}$. Twelve micrometer sections were taken from the proximal region of the hindlimb shank that contains the plantaris muscle and dried on previously subbed slides. Muscle cross-sections were double immunostained with S58 (a monoclonal IgA antibody that exclusively recognizes slow myosin heavy chain; Crow and Stockdale, 1986) (a gift kindly provided by F. E. Stockdale, Stanford University, Stanford, CA) and 5D2 (a IgG monoclonal antibody that exclusively recognizes fast muscle fiber sarcoplasmic reticulum $\mathrm{Ca}^{2+}$ ATPase; Kaprielian and Fambrough, 1987) (a gift kindly provided by D. Fambrough, The John Hopkins University, Baltimore, MD). Because the S58 antigen is sensitive to aldehyde fixation, muscle sections were incubated with S58 for $1.5 \mathrm{hr}$ (room temperature), washed several times with PBS, and then fixed for $20 \mathrm{~min}$ in $3.7 \%$ formaldehyde-PBS (room temperature). After the wash in PBS, they were incubated for $1.5 \mathrm{hr}$ (room temperature) with 5D2, washed in PBS, and incubated overnight $\left(4^{\circ} \mathrm{C}\right)$ in a secondary antibody cocktail containing goat anti-mouse fluorescein-conjugated secondary antibody (IgA; Cappell, West Chester, PA) and goat anti-mouse tetramethylrhodamine-conjugated secondary antibody (IgG; Sigma). The sections were then washed several times in PBS and finally mounted in $50 \%$ glycerin-PBS containing $0.03 \mathrm{mg} / \mathrm{ml}$ $p$-phenylenediamine.

SDS-PAGE immunoblotting. Posterior ilifibularis (PITIB) and external adductor (ADD) muscles were carefully dissected from St. 36 and 38 chick and quail hindlimbs. The muscles were suspended and mechanically homogenized in extraction buffer (50 mM HEPES, $150 \mathrm{~mm} \mathrm{NaCl}, 1$ mM EDTA, 2 mM PMSF, $100 \mu \mathrm{m}$ leupeptin, $0.2 \mathrm{TIU} / \mathrm{ml}$ aprotinin, and $1 \% \mathrm{NP}-40$ ), placed on ice for $1 \mathrm{hr}$, and centrifuged for $1 \mathrm{hr}$ at $100,000 \times$ $g\left(4^{\circ} \mathrm{C}\right)$. The concentration of total solubilized protein was determined using the BCA method (Pierce, Rockford, IL) and adjusted to $500 \mu \mathrm{g} / \mathrm{ml}$. Some aliquots $(20 \mu \mathrm{l})$ were treated with neuraminidase $(5 \mu \mathrm{l} ; 0.2 \mathrm{U} / \mathrm{ml}$ of Vibrio Cholerae; Calbiochem-Behring, San Diego, CA) for 2-3 hr at $37^{\circ} \mathrm{C}$ to remove sialic acid, and other aliquots from the same sample were treated with buffer $(5 \mu l)$ alone. SDS sample buffer containing dithiothreitol was added to each sample and heated for $20 \mathrm{~min}$ at $65^{\circ} \mathrm{C}$, and then the proteins were separated by SDS-PAGE (Laemmli, 1970) on a $6 \%$ gel. Twenty microliters of total protein (i.e., $10 \mu \mathrm{g} / \mathrm{lane}$ ) was loaded in each lane. Proteins were transferred to Immobilon-P membranes (Millipore, Bedford, MA), placed in TBS containing 4\% milk for $1 \mathrm{hr}$, and incubated overnight $\left(4^{\circ} \mathrm{C}\right)$ in $5 \mathrm{E}$ diluted in TBS and $1 \%$ milk. $5 \mathrm{E}$ is a monoclonal $\mathrm{IgG}$ antibody that recognizes all isoforms of NCAM (Frelinger and Rutishauser, 1986). Membranes were washed several times with TBS and then incubated for $1 \mathrm{hr}$ with alkaline phosphataseconjugated mouse IgG secondary antibody (1:500; Sigma). Bands were visualized using the 5-brom-4-chlor-indolyl-phosphate/nitrobluetetrazolium-chloride method.

\section{RESULTS}

Previous studies from this laboratory showed that motoneurons innervating developing fast muscle fibers express significantly more PSA than those innervating slow fibers (Landmesser et al., 1990). Enzymatic removal of PSA from both motoneurons and muscle fibers during the initial period of muscle innervation dramatically changes the branching pattern of the fast motoneurons to a slow pattern (Landmesser et al., 1990). Although we hypothesized that this alteration in branching pattern was caused by the loss of PSA on the innervating fast motoneurons, we could not exclude the possibility that fast and slow muscle fibers express different levels of PSA and that this, in turn, determines fast and slow intramuscular nerve branching patterns.

Both PSA and NCAM isoforms are regulated in temporally and spatially complex patterns on developing myotubes (Covault et al., 1986; Fredette et al., 1993; Yoshimi et al., 1993; Rafuse and Landmesser 1996). However, to date no studies have examined whether developing fast and slow muscle fibers differentially regulate NCAM isoforms or PSA. To address this issue, SDS-PAGE, combined with immunoblot analysis, was performed on muscle homogenates collected from muscles containing only slow (the ADD) or fast myotubes (the PITIB) at two different stages of development. Half of the muscle homogenates were treated with neuraminidase to remove all of the sialic acid to visualize individual NCAM isoforms. Previous studies have shown that the predominant NCAM isoforms expressed by developing myotubes are the 130, 145, and $155 \mathrm{kDa}$ NCAM isoforms (Fredette et al., 1993; Yoshimi et al., 1993; Rafuse and Landmesser, 1996). During muscle development, there is a gradual shift in the level of expression of individual NCAM isoforms such that, early in development, the 145 and $155 \mathrm{kDa}$ isoforms are the most highly expressed, whereas the $130 \mathrm{kDa}$ isoform becomes the most prominent isoform late in myogenesis (Fredette et al., 1993; Yoshimi et al., 1993). In agreement with these previous studies, both fast (Fig. 1A, lane 1) and slow (Fig. 1A, lane 3) muscles express 


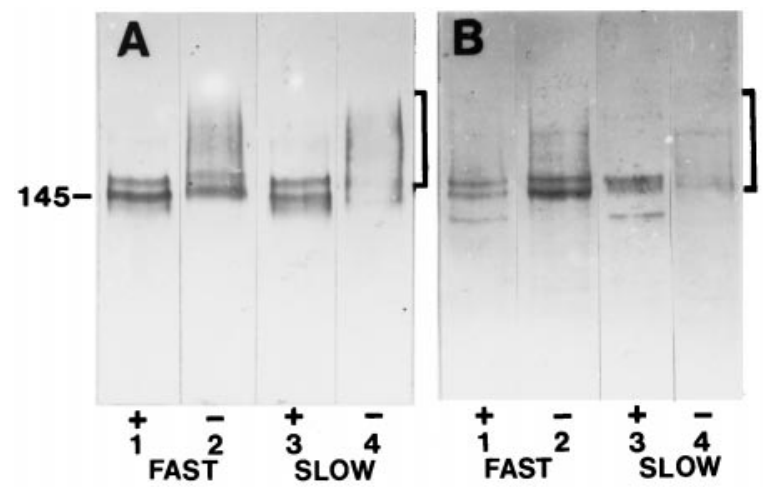

Figure 1. SDS gel electrophoresis and immunoblot analysis of NCAM isoforms and degree of polysialylation of fast and slow muscle fibers during two stages of development. $A$, Neuraminidase-desialylated proteins (+ lanes) from both developing fast (lane 1) and slow (lane 3) muscles at St. 36 indicate that muscles at this stage express the 145 and $155 \mathrm{kDa}$ NCAM isoforms, whereas the $130 \mathrm{kDa}$ isoform is barely detectable. The relative level of expression of the NCAM isoforms does not differ significantly between developing fast and slow muscles. In contrast, fast muscles at St. 36 (lane 2) express significantly more PSA, which is detected as a diffuse band running between 250 and $140 \mathrm{kDa}$ (-lanes; brackets) compared with developing slow (lane 4) muscles at the same stage of development. However, the presence of discrete bands in the non-neuraminidase-treated samples at $\sim 145$ and $155 \mathrm{kDa}$ (lane 2) indicates that a portion of 145 and $155 \mathrm{kDa}$ NCAM isoforms are not polysialylated in developing fast muscles. In contrast, the absence of these bands in slow muscles at St. 36 (lane 4 ) indicates that all isoforms are, to the some degree, sialylated in slow muscle. $B$, Neuraminidase-desialylated proteins (+ lanes) from developing fast (lane 1) and slow (lane 3) St. 38 muscles shows that the relative level of the $130 \mathrm{kDa}$ NCAM isoform is upregulated compared with the 145 and $155 \mathrm{kDa}$ isoforms by this stage in both fast and slow muscles. As observed at St. 36, fast muscles (lane 2) contain more PSA than slow muscles (lane 4) as indicated by the darker band between 250 and $140 \mathrm{kDa}$. In addition, a portion of the 145 and 155 $\mathrm{kDa}$ NCAM isoforms remains unpolysialylated in the developing fast muscles (lane 2) compared with slow muscles (lane 4 ).

primarily the 145 and $155 \mathrm{kDa}$ NCAM isoform at St. 36. Later in development, at St. 38, the expression of the $130 \mathrm{kDa}$ NCAM isoform increases, relative to the other two major isoforms, in both fast (Fig. 1B, lane 1) and slow (Fig. 1B, lane 3) myotubes (see also Fredette et al., 1993). Although the relative level of expression of the individual NCAM isoforms is not dramatically different between fast and slow myotubes at the different stages of development, PSA is differentially expressed by fast and slow developing muscle fibers. When PSA is expressed by myotubes, it appears as a diffuse band between $250-140 \mathrm{kDa}$ on immunoblots stained with an antibody that recognizes all NCAM isoforms. As shown in Figure $1 A$, the fast PITIB muscle (lane 2) has significantly more PSA than the all slow ADD muscle (Fig. 1 $A$, lane 4 ). The appearance of discrete bands in the untreated fast muscle homogenates (Fig. 1A,B, lane 2), but not in the slow muscle homogenates (Fig. $1 A, B$, lane 4 ), indicates that at least some of the 145 and $155 \mathrm{kDa}$ NCAM isoforms are not polysialylated in developing fast muscle, whereas all are to some degree polysialylated in slow muscle. However, when the $130 \mathrm{kDa}$ isoform appears at St. 38 (Fig. 1B, lanes 2, 4), it is completely polysialylated in both slow and fast muscles and only appears after removal of PSA (Fig. 1B, lanes 1, 3). At present, it is not known what factors account for these differences in degree of sialylation of the NCAM isoforms between fast and slow muscle.

In summary, developing fast muscle expresses significantly more PSA than slow muscle during the period of innervation and
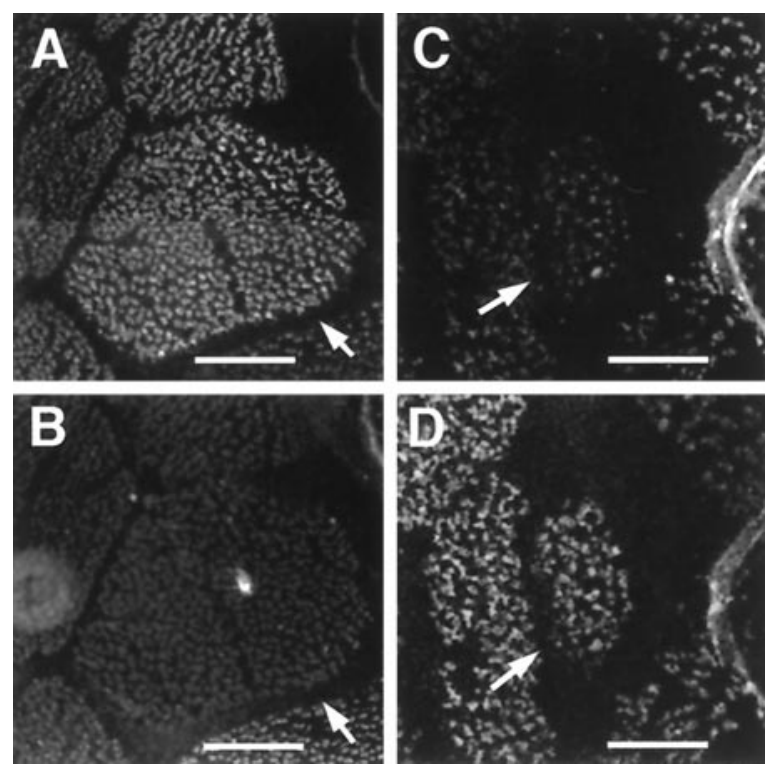

Figure 2. Double immunostaining for slow muscle myosin (with S58) and fast muscle ATPase (with 5D2) shows that, during early neuromuscular development, the chick plantaris muscle is exclusively composed of slow muscle fibers, whereas the quail plantaris muscles contains only fast fibers. In all panels, dorsal is $u p$, and anterior is left. $A, B$, Cross-sections through a St. 36 chick shank double immunostained with S58 $(A)$ and 5D2 $(B)$ shows that, at this stage in development, the chick plantaris muscle (indicated by arrow) contains only slow fibers. $C, D$, Cross-section through a St 36 quail shank double immunostained with slow S58 $(C)$ and fast 5D2 $(D)$ shows that early in neuromuscular development the quail plantaris muscle (indicated by arrow) is exclusively composed of fast muscle fibers. Scale bars, $100 \mu \mathrm{m}$.

subsequent intramuscular nerve branching. Whether this differential expression accounts for the dramatically different intramuscular branching patterns in fast and slow avian muscles is addressed below.

Using the myfibrillar ATPase reaction to histochemically identify different muscle fiber types, Grim et al. (1989) showed that, late in development (i.e., St. 45), the chick plantaris muscle is exclusively composed of multiply innervated tonic type IIIA and IIIB (i.e., slow fibers) muscle fibers. Acetylcholinesterase staining of whole muscle teased fiber preparations showed that the tonic fibers were indeed multiply innervated along their length (Barnard et al., 1982; Grim et al., 1989). In contrast, the late embryonic quail plantaris muscle contains both focally innervated type II fibers (i.e., fast) and multiply innervated type IIIA and IIIB fibers (Grim et al., 1989).

The muscles described above, at the end of embryonic development, are composed primarily of "secondary" muscle fibers that are generated during the last half of embryonic development [embryonic day 10 (E10) to E20]. However, the only fibers present in developing muscle during initial innervation (St. 30-36; i.e., E6-E10), when the nerve branching patterns are being established, are primary myofibers. Because only these myofibers could be affecting the initial pattern of innervation, we characterized the fiber type distribution of St. 35 (E8-E9) chick and quail muscles. We immunostained chick and quail plantaris muscles with two antibodies that specifically recognize slow or fast muscle fibers (see Materials and Methods for more detail). Figure 2 (arrows) shows the distribution of slow (Fig. 2A) and fast (Fig. $2 B$ ) muscle fibers in chick, showing that the chick plantaris muscle at this developmental stage is entirely composed of slow 
fibers. In contrast, the quail plantaris muscle at this stage is entirely composed of fast fibers (Fig. 2D) and contains no slow fibers (Fig. 2C). Supporting the study by Grim et al., (1989), we found that, much later in development (E17), the chick plantaris muscle remained exclusively composed of slow fibers (Fig. 3A), whereas the quail plantaris muscle came to contain populations of both slow and fast fibers (Fig. $3 B$ ). Single, rhodamine-conjugated $\alpha$-bungarotoxin-labeled muscle fibers teased from St. 43 chick and quail plantaris muscles confirmed that all chick plantaris fibers (Fig. $3 E$ ) contained multiple endplates (i.e., slow), whereas quail plantaris muscle was composed of fibers that had either single (fast) (Fig. 3C) or multiple (slow) (Fig. 3D) endplates.

Previous studies have shown that the pattern of intramuscular nerve branching is dramatically different in slow and fast muscles (Dahm and Landmesser 1988; Landmesser et al., 1990, Rafuse et al., 1996). The main intramuscular nerve branches innervating slow muscle grow parallel to the muscle fibers and extend many small collateral sprouts at regular intervals to multiply innervate single muscle fibers along their entire length (Fig. 4B; muscle fibers in this and all subsequent figures run from left to right). In contrast, the main nerve branches innervating fast muscles grow transverse to the fibers and undergo a characteristic reductive branching pattern in which each subsequent branch is smaller in diameter than the previous one (Fig. 4D). This pattern of branching ensures that a single fast motor axon can branch and focally innervate a large number of fast muscle fibers.

Whole mounts of early (Fig. 4A,C) and late St. 35 (Fig. 4B,D) chick and quail plantaris muscles clearly show that the pattern of intramuscular nerve branching differs dramatically in these two species of birds. Because chick plantaris muscles are homogeneously composed of slow fibers, the main nerve branches grow parallel to the fibers at the very onset of muscle innervation (Fig. $4 A$ ) (early St. 35), and this basic pattern remains as the muscle grows and additional branches are formed (Fig. 4B) (late St. 35). In contrast, early developing quail muscles contain only fast fibers, and consequently the main nerve branches grow transverse to the fibers and undergo the characteristic reductive nerve branching pattern (Fig. $4 C$ ). This pattern is maintained as additional branches form by late St. 35 (Fig. 4D)

To determine what type of a branching pattern slow chick motoneurons would form when innervating fast quail muscle, we replaced chick hindlimb buds with quail hindlimb buds at St 18 . This stage is before when motoneurons exit the spinal cord. Results from a previous study had shown that quail hindlimb muscle motoneuron pools are located in the same relative spinal cord position as the homologous chick motoneuron pools (Tanaka and Landmesser, 1986a). Furthermore, these studies showed that, in chick-quail hindlimb chimeras, motoneuron pools from one species were found to selectively innervate the homologous muscles in the other species with remarkable precision (Tanaka and Landmesser, 1986a), although the relative composition of muscle fiber types in the two species is not identical. These results indicate that limb-derived guidance cues required for motoneurons to innervate their appropriate muscles override those cues guiding them to innervate their appropriate muscle fiber type (Rafuse et al., 1996). In addition, several studies have shown that the basic patterning of avian fast and slow myotubes within muscles is an autonomous property of the limb and does not require neuronal input or activity to initially develop (Butler et al., 1982; Fredette and Landmesser, 1991b). Finally, it has been shown that the initial pattern of fast-slow myotube distribution in chick-quail chimeras is also autonomous to the implanted limb (Tanaka and Landmesser, 1986b; our unpublished observations).

Based on the above observations, we were able to use the chick-quail hindlimb chimera as a model to directly address the question as to whether the pattern of intramuscular nerve branching in the plantaris muscle is determined by some property of the innervating motoneurons or caused by some intrinsic property of the innervated muscle fibers (i.e., fast vs slow). If some property of the motoneuron determines the intramuscular branching pattern, slow chick plantaris axons should branch in a characteristic slow pattern (Fig. $5 A$ ) while innervating the all fast quail plantaris muscle in chick-quail hindlimb chimeras (Fig. 5C). Alternatively, if the branching pattern formed is the characteristic reductive pattern associated with fast muscles (Fig. $5 B$ ), one can conclude that the pattern of branching is determined by some intrinsic property of the developing fast muscle fibers (Fig. 4D).

Figure $6 A$ shows a typical example of extensive reductive intramuscular nerve branching in a normal St. 36 quail plantaris muscle. As observed in younger quail plantaris muscles, the main intramuscular branches grow transverse to the muscle fibers. In contrast, the main nerve branches innervating a St. 36 chick plantaris muscle grow parallel to the muscle fibers (Fig. 6B). When slow chick plantaris motoneurons were forced to innervate fast quail plantaris muscles in chick-quail hindlimb chimeras, the main intramuscular nerve branches appeared to first enter the muscle transversely, as characteristic of fast muscle (Fig. 6C). However, unlike the characteristic reductive branching pattern normally observed in St. 36 quail plantaris muscles (Fig. 6A), once in the muscle, the main intramuscular nerve branches turned and grew parallel to the muscle fibers (Fig. $6 C$ ) in a pattern that is very similar to that normally seen in slow avian skeletal muscles. These results strongly support the hypothesis that some intrinsic property of the innervating motoneuron determines the pattern of intramuscular nerve branching.

What property of the motoneuron might account for the differences in nerve growth and branching? In a previous study, it was shown that fast motoneurons innervating the fast region of the chick iliofibularis (IFIB) muscle express more PSA than the slow IFIB motoneurons innervating the slow region (Landmesser et al., 1990). The expression of PSA on cells decreases the degree of cell-cell adhesion, presumably because of its large size and strong negative charge (for review, see Rutishauser and Landmesser, 1996). Based on these findings, Landmesser et al. (1990) enzymatically removed PSA from both developing IFIB motoneurons and muscle fibers during early neuromuscular development and showed that the normal reductive branching pattern in the fast region was altered to more closely resemble a slow branching pattern.

To determine whether differential expression of PSA on fast and slow motoneurons determines the pattern of intramuscular nerve branching in developing chick and quail plantaris muscles, Endo-N was injected into the hindlimbs of St. 30 embryos to specifically remove PSA from the innervating axons and developing muscle fibers. As shown in the St. 36 whole mounts of the fast quail plantaris muscle from one of the Endo-N-injected quail embryos (Fig. 7B) (see Materials and Methods for details), the intramuscular nerve branching pattern was dramatically altered from the normal fast quail pattern (Fig. $7 A$ ) to resemble a normal slow pattern (Fig. $4 A, B$ ). Unlike the high degree of defasciculation, transverse pattern of axon growth, and reductive branching normally observed in St. 36 quail plantaris muscles (Fig. $7 A$ ), the axons innervating the Endo-N-treated plantaris muscles were 

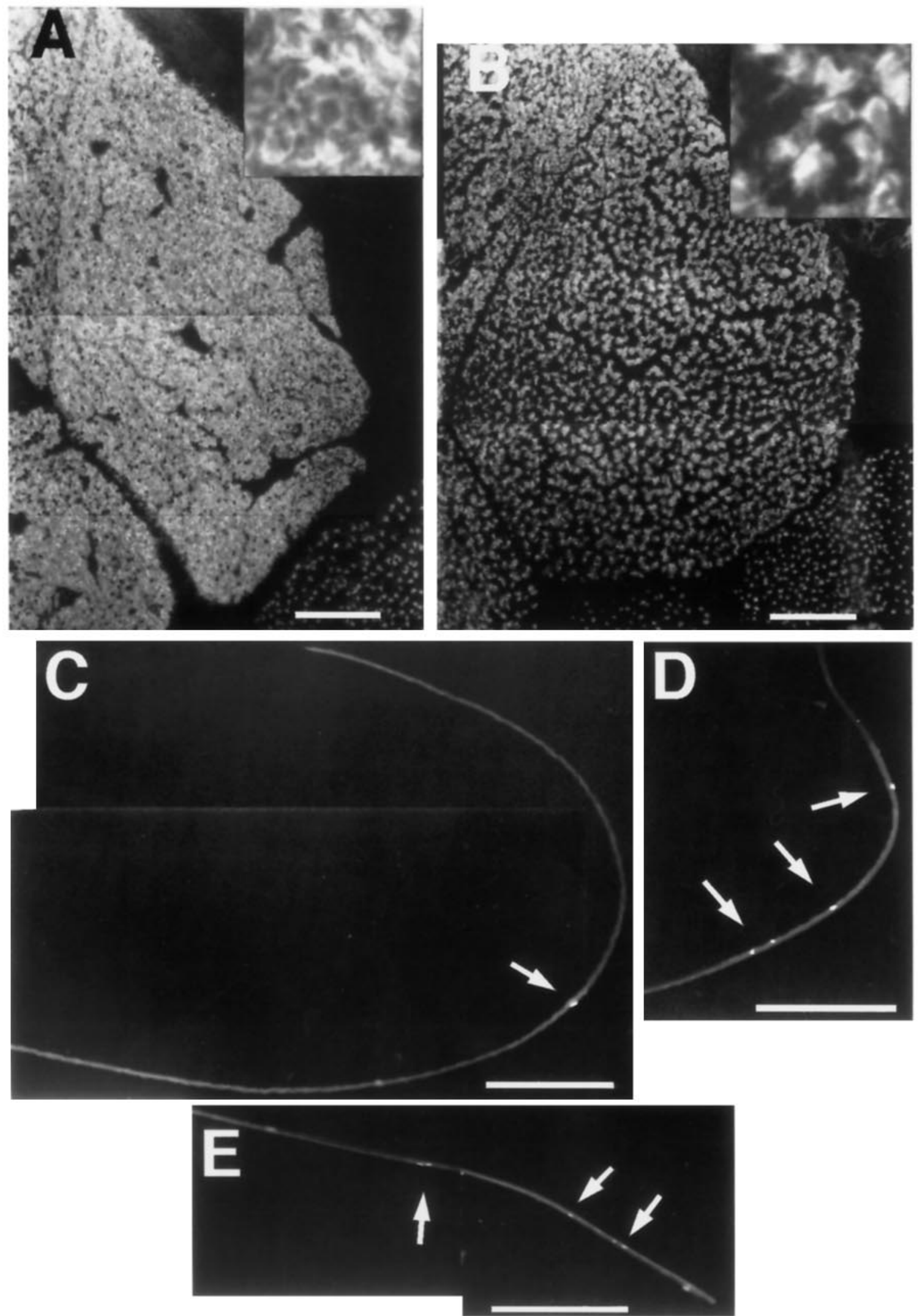

Figure 3. Late in myogenesis, the chick plantaris muscle is homogeneously composed of slow muscle fibers, whereas the quail plantaris muscle contains both fast and slow fibers. $A$, Cross-section through a St. 43 chick shank shows that all chick plantaris myotubes are S58-positive, indicating that at this late stage in development the chick plantaris muscle is homogeneously composed of slow fibers. $B$, Cross-section through a St. 43 quail shank shows that only approximately half of the plantaris myotubes are S58-positive, indicating that at this late stage in development the quail plantaris muscle is 

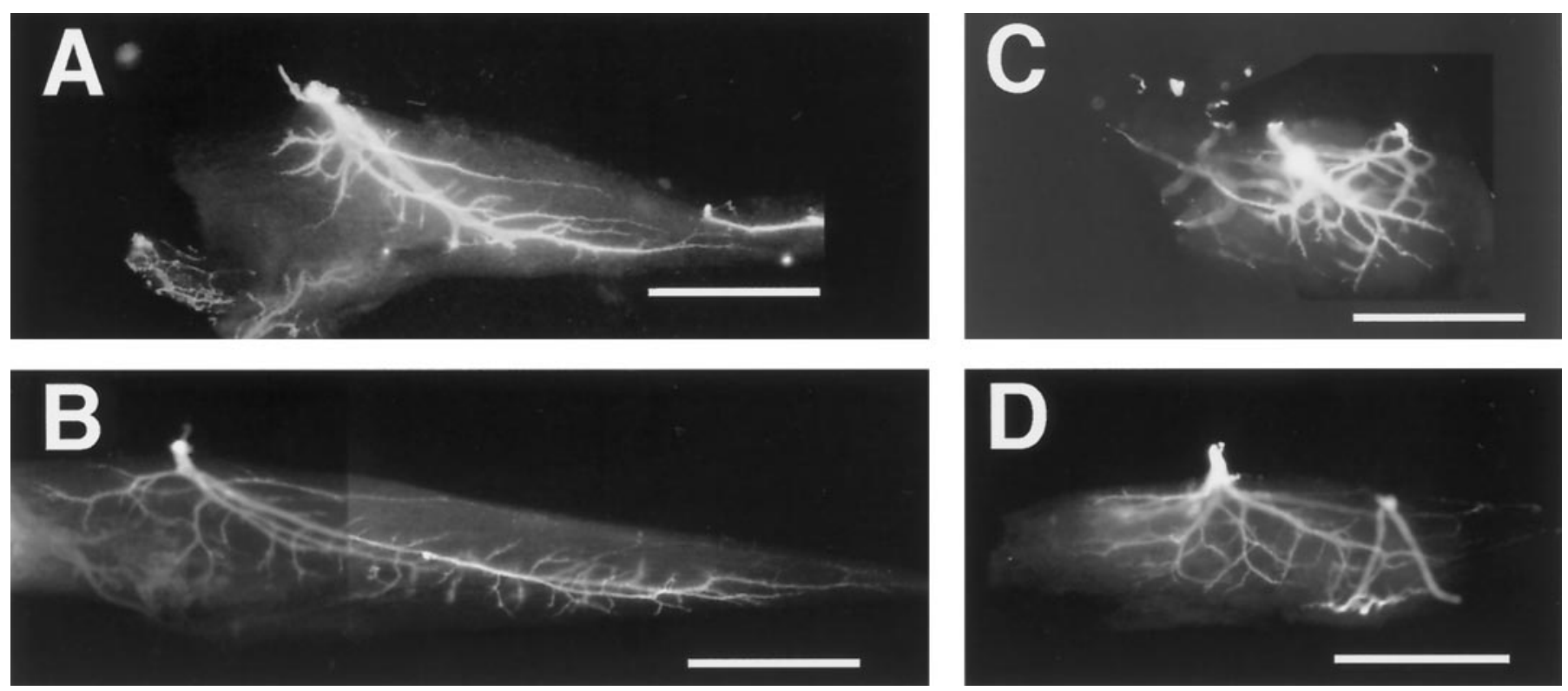

Figure 4. The distribution of intramuscular nerves in chick and quail plantaris muscles differs during early muscle development. In $A-D$, the muscle fibers run from left (proximal) to right (distal). $A, B$, In the slow chick plantaris muscle, at an early St. 35 , the nerve enters the muscle proximally, and the main intramuscular branches are tightly fasciculated together forming main nerve trunks that grow parallel to the myotubes $(A)$. During late St. 35 , the main chick plantaris intramuscular nerves remain tightly fasciculated but begin to extend short collateral sprouts intermittently along their length $(B)$. $C, D$, In the fast quail plantaris muscle, at an early St. 35, the main intramuscular nerve enters the muscle at its midpoint, and the branches grow transversely across the muscle fibers and undergo reductive branching $(C)$. By late St. 35 , the main nerve branches continue to grow predominantly transverse to the myotubes and begin to branch further in a reductive pattern $(D)$. Scale bars, $500 \mu \mathrm{m}$.

highly fasciculated, did not undergo reductive branching, and grew parallel to the muscle fibers (Fig. 7B). All these patterns of growth are characteristics of a slow pattern. As a control, removal of PSA from slow chick plantaris muscles did not alter the basic branching pattern, which remained slow in nature (Fig. 7D) and similar to normal chick plantaris (Fig. 7C). However, the number of secondary branches that emerged from the main nerve trunks was clearly reduced compared with control. This is not unexpected because PSA is still present on slow nerve branches, although at lower levels than fast. This observation is also consistent with an earlier study that found a reduction in the secondary branches in the slow IFIB muscle after complete removal of PSA with Endo-N (Landmesser et al., 1990).

Together, these results strongly indicate that the intramuscular nerve branching pattern is determined by the degree of polysialylation of the innervating fast and slow motoneurons.

\section{DISCUSSION}

Neurons innervating avian fast and slow muscles, or muscle regions, have dramatically different intramuscular branching patterns, which in turn determine the different patterns of synapse formation observed on fast and slow myotubes (Dahm et al., 1988, 1991). The results presented here extend our previous studies, which showed that the pattern of branching in slow and fast muscle was strongly modulated by PSA (Landmessser et al., 1990). Here, we show that the characteristic reductive branching pattern of fast motor axons is caused by a high level of PSA expression on the innervating neurons, whereas slow axons exhibit the more fasciculated slow pattern attributable to low PSA expression. Furthermore, the present study makes the novel finding that neither the architectural structure of developing myotubes nor the distinct molecular make-up of developing fast and slow muscle fibers regulates intramuscular branching patterns. Thus, slow chick plantaris motoneurons innervating fast quail plantaris muscles in chick-quail hindlimb chimeras displayed a characteristic slow branching pattern. This indicates that the pattern is regulated by some property of the motor axons and is not a result of some structural or molecular property of the fast fibers being innervated. In addition, enzymatic removal of PSA from fast motoneurons altered the normal reductive branching pattern to a highly fasciculated slow axon branching pattern, indicating that the level of PSA on the innervating axon plays a major role in regulating intramuscular axon branching.

\section{Determinants of intramuscular nerve branching}

The first generation of muscle fibers to develop are primary myotubes, which differentiate into distinct fast and slow phenotypes by a nerve-independent process. Secondary myotubes begin to develop alongside the primary myotubes, in what is thought to be a nerve-dependent process, only after the muscle has been innervated by all of its motoneurons (Butler et al., 1982; Phillips and Bennett, 1984; Crow and Stockdale, 1986; Condon et al.,

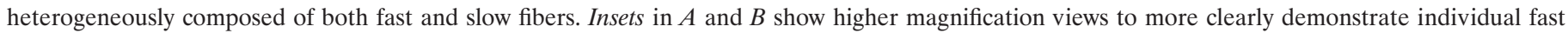

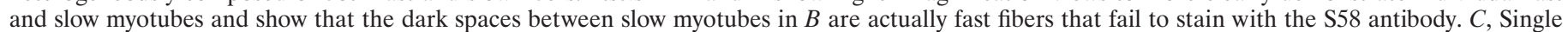

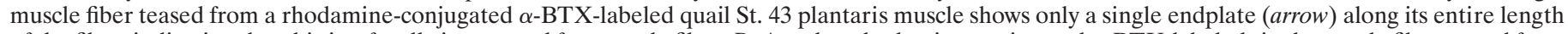

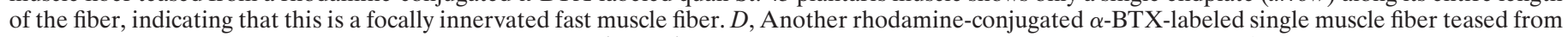

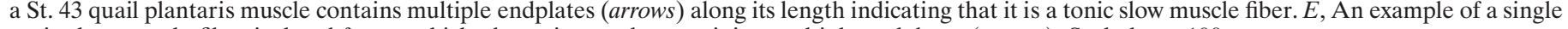
tonic slow muscle fiber isolated from a chick plantaris muscle containing multiple endplates (arrows). Scale bars, $100 \mu \mathrm{m}$. 


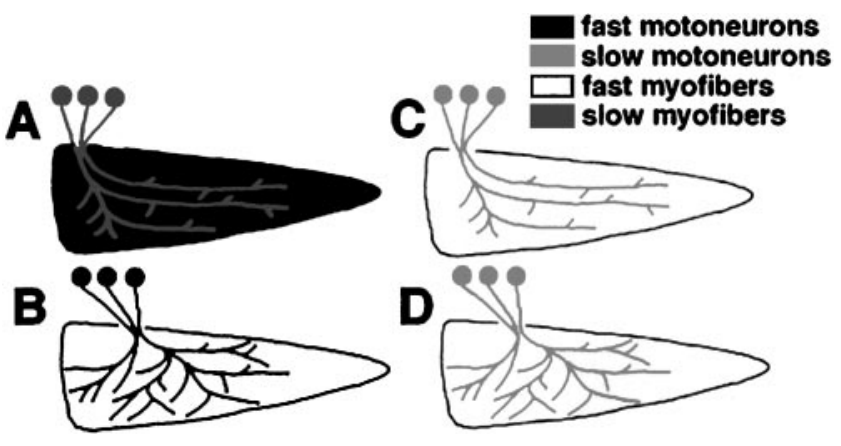

Figure 5. Schematic representation of the chick-quail hindlimb experimental model and intramuscular nerve branching hypothesis. $A$, In normal chick plantaris slow muscles, the main intramuscular nerve branches grow parallel to the muscle fibers and extend small collateral sprouts at regular intervals. $B$, In normal quail fast plantaris muscles, the main intramuscular nerve branches grow transversely to the myotubes and undergo characteristic reductive branching. $C$, Proposed plantaris intramuscular nerve branching pattern in the chick-quail hindlimb chimera if some property of the slow chick plantaris motoneurons regulates the branching pattern, although the axons are innervating a fast quail plantaris muscle. $D$, Proposed plantaris intramuscular nerve branching pattern in the chick-quail plantaris muscle if the branching pattern is regulated by some property of the fast quail plantaris myotubes.

1990; Fredette and Landmesser, 1991a,b). The basic fast-slow intramuscular nerve branching patterns are established before the onset of secondary myogenesis (Dahm and Landmesser, 1988) at a time when few synapses have formed and the muscle is only beginning to be activated (Dahm and Landmesser, 1991). In previous studies, we proposed that intramuscular nerve branching patterns were regulated by the relative strength of axon-axon versus axon-myotube adhesion (Landmesser et al., 1988, 1990). According to this model, increasing axon-axon adhesion would promote axon fasculation, resulting in a slow branching pattern such that the main intramuscular branches grow parallel to the muscle fibers. Decreasing axon-axon adhesion, while simultaneously increasing axon-myotube adhesion, would promote a fast pattern of axon branching such that the axons grow transverse to the myotubes and undergo a reductive branching pattern. In the chick IFIB muscle, the axons innervating fast myotubes express significantly more PSA than axons innervating slow myotubes (Landmesser et al., 1990). Enzymatic removal of PSA from both axons and myotubes, at the time when the IFIB muscle is first being innervated, dramatically alters the normal branching pattern such that the axons innervating the fast region now grow with a slow branching pattern (Landmesser et al., 1988, 1990).

These results raised the possibility that intramuscular branching patterns are regulated by the level of PSA expressed by innervating neurons. However, developing myotubes also express PSA in a developmentally regulated manner (Fredette et al., 1993), as we have also shown in the present study. In addition, we demonstrated that developing fast muscles express higher levels of PSA than slow muscle (Fig. 1). Consequently, our previous study could not determine whether it was PSA levels on motoneurons or muscle fibers that influenced the intramuscular branching pattern. By forcing slow chick neurons to innervate a fast quail muscle in chick-quail hindlimb chimeras, we are now able to demonstrate that the intramuscular branching pattern is regulated by the level of PSA being expressed by the innervating neurons. In addition, enzymatic removal of PSA during early plantaris nerve innervation dramatically altered the fast nerve
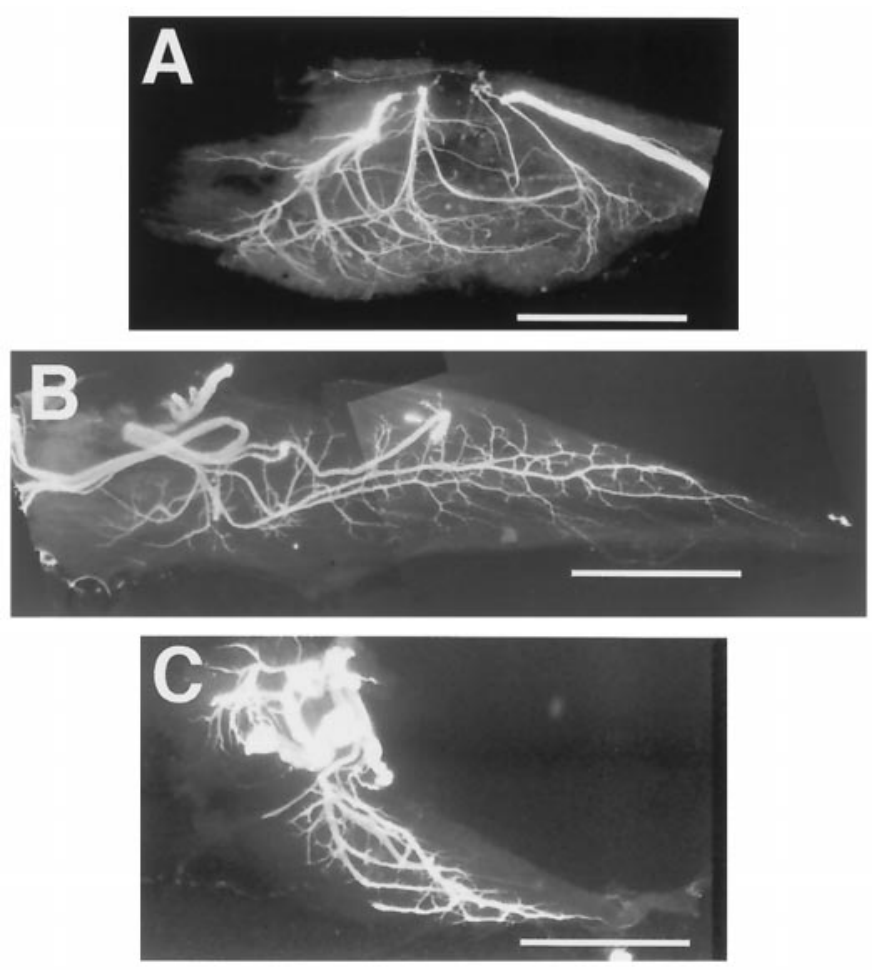

Figure 6. The intramuscular nerve branching pattern is determined by some property of the innervating motoneurons. $A$, Intramuscular nerve branching pattern in a normal quail plantaris at St. 36 showing that the main nerve trunks grow transversely across the myotubes and undergo characteristic reductive branching. $B$, Intramuscular nerve branching pattern in a normal chick plantaris muscle at St. 36 showing that the main nerve trunks are highly fasciculated and grow parallel to the myotubes. $C$, When slow chick plantaris motoneurons are forced to innervate a fast quail plantaris muscle in a chick-quail hindlimb chimera, the axons grow in a tightly fasciculated pattern and grow parallel to the myotubes. This pattern of branching is dramatically different than the pattern seen in normal quail plantaris muscles and more closely resembles the pattern observed in normal slow chick plantaris muscle. Scale bars, $500 \mu \mathrm{m}$.

branching pattern such that it more closely resembled a slow pattern. Removal of PSA from developing slow chick plantaris muscles produced only moderate changes in the branching pattern. Together, these results indicate that the intramuscular branching pattern is regulated by the level of PSA expressed by the innervating neurons and is independent of the type of muscle fiber being innervated.

Previous studies have shown that muscle fibers can change their phenotype late in development (i.e., St. 41-42) if their pattern of neuromuscular activity is experimentally altered for a number of days by either chronic $d$-tubocurarine neuromuscular blockade (Gauthier et al., 1984), spinal cord electrical stimulation (Toutant et al., 1979), or foreign motoneuron innervation (Vogel and Landmesser, 1987; Grim et al., 1989). Consequently, we cannot rule out the possibility that conversion of muscle fiber properties later in development might have modified late stages of the intramuscular branching pattern. However, such activitydependent conversion of fiber types cannot have influenced the initial branching patterns that we characterized, because these patterns would have been formed before synapse formation and the electrical activation of most of the muscle fibers (Dahm and Landmesser, 1991). 

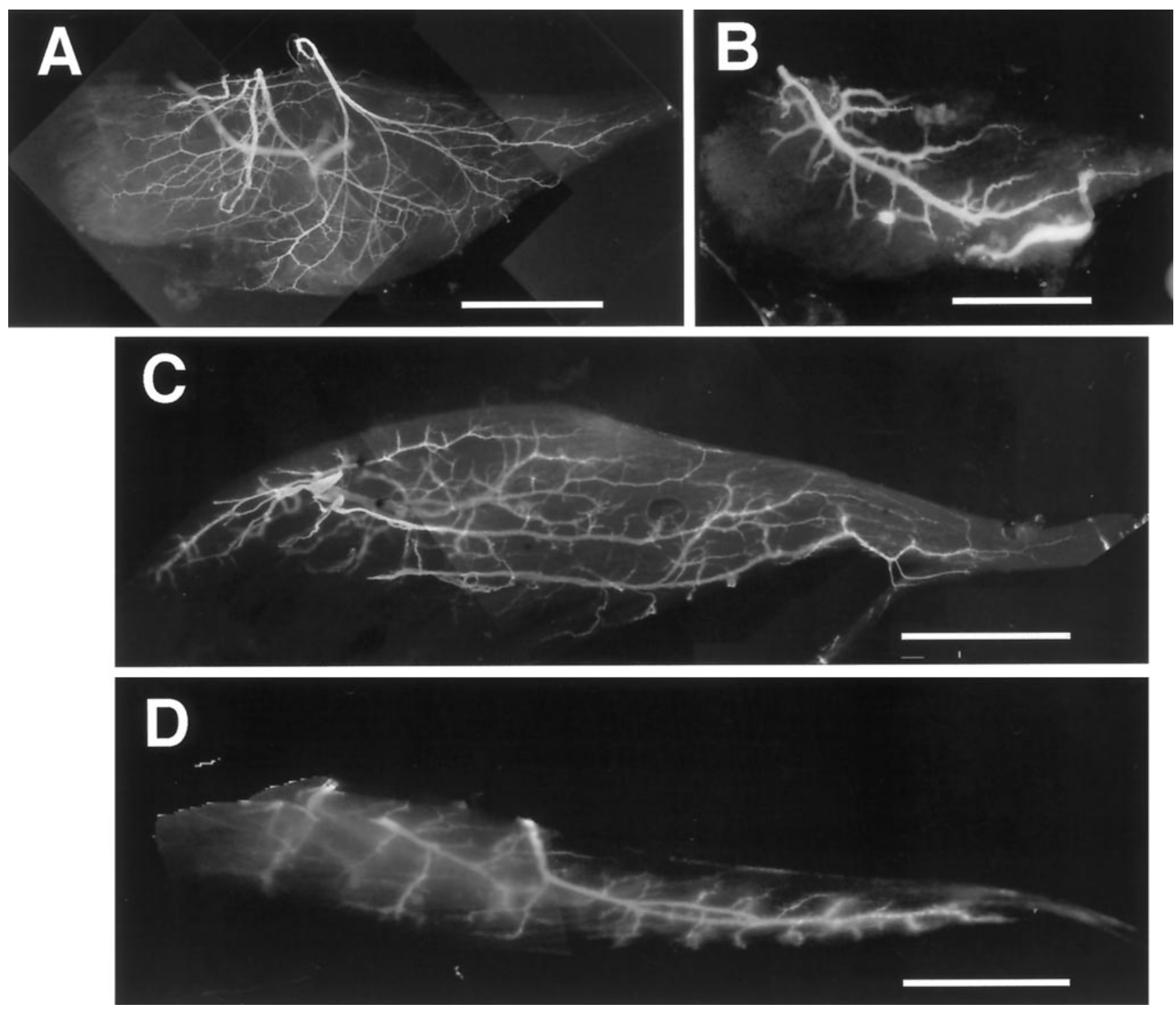

Figure 7. The intramuscular slow nerve branching pattern is determined by the level of PSA on the innervating motoneurons. $A$, Intramuscular nerve branching in late St. 36 normal quail plantaris muscle. $B$, Enzymatic removal of PSA from normal quail plantaris nerves and muscles during the period of initial muscle innervation dramatically alters the pattern of innervation such that the nerves become highly fasciculated with the major intramuscular nerve growing parallel to the myotubes. $C$, Intramuscular nerve branching pattern in a late St. 36 normal chick plantaris muscle. $D$, Enzymatic removal of PSA from normal chick plantaris nerve and muscle during the period of initial innervation does not dramatically alter the branching pattern, although there is some reduction in the number of secondary branches that emerge from the main nerve trucks. Scale bars, $500 \mu \mathrm{m}$.

\section{Regulation of PSA during development}

PSA expression is regulated on developing axons and myotubes in complex spatial and temporal patterns (Rutishauser and Landmesser, 1996). How such regulation occurs is not well understood, but two sialyltransferases that can add PSA to NCAM have been characterized (Kojima et al., 1995; Nakayama et al., 1995; Yoshiba et al., 1995; Ong et al., 1998). Contact with muscle targets results in a decrease in PSA levels and polysialyltransferase activity in ciliary ganglion neurons (Bruses et al., 1995). Electrical activity has also been shown to regulate PSA expression on chick motor axons and myotubes. Blocking muscle fiber electrical activity, both in vivo (Fredette et al., 1993) and in culture (Rafuse and Landmesser, 1996), results in decreased PSA expression on developing myotubes. Similarly, increasing muscle fiber activity increases PSA on developing myotubes in culture (Rafuse and Landmesser, 1996). Additionally, it has been found recently that in vivo application of the neuromuscular blocking agent $d$-tubocurare, which was previously shown to increase PSA expression on motor axons (Landmesser et al., 1990), actually may enhance motoneuron bursting activity (Milner and Landmesser, 1999; Usiak and Landmesser, 1999). Thus, in both nerve and muscle, PSA expression may require electrical activity. How might activity be coupled to PSA expression? In muscle, the activity-dependent synthesis of PSA was shown to require influx of $\mathrm{Ca}^{2+}$ through voltage-activated $\mathrm{Ca}^{2+}$ channels and subsequent activation of PKC (Rafuse and Landmesser, 1996). More recently, Bruses and Rutishauser (1998) showed that PSA synthesis in chick ciliary ganglion and optic tectum is coupled to the concentration of $\mathrm{Ca}^{2+}$ in intracellular compartments, and polysialylation of NCAM only occurs if $\mathrm{Ca}^{2+}$ is within a certain range (0.1-1.0 mM).

We have shown here that PSA on motor axons regulates the 
fast-slow pattern of intramuscular nerve branching, and previous studies have shown that fast and slow motor axons have different levels of PSA. Is electrical activity only permissive for PSA expression, or could different patterns of electrical activity in fast and slow motor axons explain their differential PSA expression? Developing chick flexor and extensor motoneurons have been shown to be spontaneously active early in development (several days before nerve-muscle contact) and to have different patterns of activity (Milner and Landmesser, 1999). Whether fast and slow motoneurons have intrinsic differences in electrical activity or in the expression of voltage-activated $\mathrm{Ca}^{2+}$ channels (McCobb et al., 1989), which in turn could regulate PSA in an activitydependent manner, is being investigated.

\section{Possible relevance to mammalian development}

A fundamental rule in motor control is that motor units (defined as the motoneuron and all of the muscle fibers it innervates) are recruited from the least forceful to the most forceful during muscle contraction (Henneman and Olson, 1965). This orderly recruitment according to size allows for a smooth gradation of muscle force as additional units are activated (Henneman and Mendell, 1981). One reason why larger units (which tend to be fast) generate more force than smaller units (which tend to be slow) is because they branch and innervate more muscle fibers (Bodine et al., 1987; Totosy de Zepetnek et al., 1992), and such differences are already established by birth. Whether differences in the level of PSA expression by fast and slow mammalian motoneurons regulates their degree of intramuscular axon branching is unknown. However, this hypothesis is currently being tested in the muscles of NCAM null (and thus PSA null) mice, which, if the hypothesis is true, would be expected to exhibit a reduction in the range in motor unit force, because the larger motoneurons would branch less.

In summary, by using chick-quail hindlimb chimeras to force slow axons to innervate a fast muscle, we have shown that the distinct patterns of intramuscular nerve branching are determined by the type of innervating motor axon and not by the molecular properties or structure of the type of muscle fiber. Together with the results of PSA removal, this study supports the model that the pattern of intramuscular axon branching is determined by the relative amount of axon-axon versus axon-myotube adhesion and that this is regulated by the level of PSA being expressed by the developing motoneurons.

\section{REFERENCES}

Barnard FA, Lyles JM, Pizzy JA (1982) Fibre types in chicken skeletal muscles and their changes in muscular dystrophy. J Physiol (Lond) 331:333-354.

Bodine SC, Roy RR, Eldred E, Edegerton VR (1987) Maximal force as a function of anatomical features of motor units in cat tibialis anterior. J Neurphysiol 57:1730-1745.

Brown MC, Jansen JKS Van Essen D (1976) Polyneuronal innervation of skeletal muscle in new-born rats and its elimination during maturation. J Physiol (Lond) 261:387-422.

Bruses JL, Rutishauser U (1998) Regulation of neural cell adhesion molecule polysialylation: evidence for nontranscriptional control and sensitivity to an intracellular pool of calcium. J Cell Biol 140:1177-1186.

Bruses JL, Oka S, Rutishauser U (1995) NCAM-associated polysialic acid on ciliary ganglion neurons is regulated by polysialyltransferase levels and interaction with muscle. J Neurosci 15:8310-8319.

Butler J, Cosmos E, Brierley J (1982) Differentiation of muscle fiber types in aneurogenic brachial muscles of the chick embryo. J Exp Zool 224:65-80.

Condon K, Silberstein L, Blau HM, Thompson WJ (1990) Differentia- tion of fiber types in aneural musculature of the prenatal rat hindlimb. Dev Biol 138:275-295.

Couvalt J, Merlie, JP, Goridis C, Sanes JR (1986) Molecular forms of $\mathrm{N}-\mathrm{CAM}$ and its RNA in developing and denervated skeletal muscle. J Cell Biol 102:731-739.

Crow MT, Stockdale FE (1986) Myosin expression and specialization among the earliest muscle fibers of the developing avian limb. Dev Biol 113:238-254.

Dahm L, Landmesser LT (1988) The regulation of intramuscular branching during development and following activity blockade. Dev Biol 130:621-644.

Dahm L, Landmesser LT (1991) The regulation of synaptogenesis during normal development and following activity blockade. J Neurosci 11:238-255.

Fredette BJ, Landmesser LT (1991a) Relationship of primary and secondary myogenesis to fiber type development in embryonic chick muscle. Dev Biol 143:1-18.

Fredette BJ, Landmesser LT (1991b) Reevaluation of the role of innervation in primary and secondary myogenesis in developing chick muscle. Dev Biol 143:19-35.

Fredette B, Rutishauser U, Landmesser L (1993) Regulation and activity-dependence of N-Cadherin, NCAM isoforms, and polysialic acid on chick myotubes during development. J Cell Biol 123:1867-1888.

Frelinger AL, Rutishauser U (1986) Topography of N-CAM structural and functional determinants. II. Placement of monoclonal antibody epitopes. J Cell Biol 103:1729-1737.

Gauthier GF, Ono RD, Hobbs AW (1984) Curare-induced transformation of myosin pattern in developing skeletal muscle fibers. Dev Biol 105:144-154.

Grim M, Nensa K, Christ B, Jacob HJ, Tosney KW (1989) A hierarchy of determining factors controls motoneuron innervation: experimental studies on the development of plantaris muscle (PL). Anat Embryol 180:179-189.

Hamburger V, Hamilton HL (1951) A series of normal stages in the development of the chick embryo. J Morphol 88:49-92.

Henneman E, Mendell LM (1981) Functional organization of the motoneurone pool and its inputs. In: Handbook of physiology, Sec 1, The nervous system, Vol II (Brooks VB, ed), pp 423-508. Bethesda, MD: American Physiological Society.

Henneman E, Olson CB (1965) Relation between structure and function in the design of skeletal muscles. J Neurophysiol 28:581-598.

Kaprielian Z, Fambrough DM (1987) Expression of fast and slow isoforms of the $\mathrm{Ca}^{2+}$-ATPase in developing chick skeletal muscle. Dev Biol 124:490-503.

Kernell D (1992) Organized variability in the neuromuscular system: a survey of task-related adaptations. Arch Ital Biol 130:19-66.

Kojima N, Tachida Y, Yoshida Y, Tsuji S (1995) Characterization of mouse ST8Sia II (STX) as a neural cell adhesion molecule-specific polysialic acid synthase. J Biol Chem 271:19457-19463.

Laemmli U (1970) Cleavage of structural proteins during the assembly of the head of bacteriophage. Nature 227:680-685.

Landmesser L, Dahm L, Schultz K, Rutishauser U (1988) Distinct roles for adhesion molecules during innervation of embryonic chick muscle. Dev Biol 130:645-670.

Landmesser L, Dahm L, Tang J, Rutishauser U (1990) Polysialic acid as a regulator of intramuscular nerve branching during embryonic development. Neuron 4:655-667.

McCobb, DP, Best PM, Beam KG (1989) Development alters expression of calcium currents in chick limb motoneurons. Neuron 2:1633-1643.

McLennan IS (1983) The development of the pattern of innervation in chicken hindlimb muscles: evidence for specification of nerve-muscle connections. Dev Biol 97:229-238.

Milner LD, Landmesser L (1999) Cholinergic and GABAergic inputs drive spontaneous motoneuron activity before target contact. J Neurosci 19:3007-3022.

Nakayama J, Fukuda MN, Fredette B, Ranscht B, Fukuda M (1995) Expression cloning of human polysialyltransferase that forms the polysialylated neural cell adhesion molecule present in embryonic brain. Proc Natl Acad Sci USA 92:7031-7035.

Ong E, Nakayama J, Angata K, Reyes L, Katsuyama T, Arai Y, Fukuda M (1998) Developmental regulation of polysialic acid synthesis in mouse directed by two polysialyltransferases, PST and STX. Glycobiology 8:415-424.

Ono K, Tomasiewicz H, Magnuson T, Rustishauser U (1994) N-CAM 
mutation inhibits tangential neuronal migration and is phenocopied by enzymatic removal of polysialic acid. Neuron 13:595-609.

Phillips WD, Bennett MR (1984) Differentiation of fiber types in wing muscle during embryonic development: effect of neural tube removal. Dev Biol 106:457-468.

Rafuse VF, Landmesser (1996) Contractile activity regulates isoform expression and polysialylation of NCAM in cultured myotubes: involvement of $\mathrm{Ca}^{2+}$ and protein kinase C. J Cell Biol 132:969-983.

Rafuse VF, Landmesser (1997) The pattern of intramuscular nerve branching is determined by the innervating motoneuron and its level of polysialic acid (PSA). Soc Neurosci Abstr 24:767.

Rafuse VF, Milner L, Landmesser LT (1996) Selective innervation of fast and slow muscle regions during early chick neuromuscular development. J Neurosci 16:6864-6877.

Rutishauser U, Landmesser LT (1996) Polysialic acid in the vertebrate nervous system: a promoter of plasticity in cell-cell interactions. Trends Neurosci 19:422-427.

Tanaka H, Landmesser LT (1986a) Interspecies selective motoneuron projection patterns in chick-quail chimeras. J Neurosci 6:2880-2888.

Tanaka H, Landmesser LT (1986b) Cell death of lumbosacral motoneurons in chick, quail, and chick-quail chimera embryos: a test of the quantitative matching hypothesis of neuronal cell death. J Neurosci 6:2889-2899.

Totosy de Zepetnek JE, Zung H, Erdebil S, Gordon T (1992) Innervation ratio is an important determinant of force in normal and reinnervated rat tibialis anterior muscles. J Neurophysiol 67:1386-1403.

Toutant JP, Toutant MN, Renaud D, Le Douarin GH (1979) Enzymatic differentiation of muscle fibre types in embryonic Latissimus Dorsii of the chick: effects of spinal cord stimulation. Cell Differ 8:375-382.

Usiak, MF, Landmesser LT (1999) Neuromuscular activity blockade induced by muscimol and $d$-tubocrurarine differentially affects the survival of embryonic chick motoneurons. J Neurosci 19:7925-7939.

Vogel MW, Landmesser L (1987) Distribution of fiber types in embryonic chick limb muscles innervated by foreign motoneurons. Dev Biol 119:481-495

Yoshiba Y, Kojima N, Tsuji S (1995) Molecular cloning and characterization of a third type of $\mathrm{N}$-glycan 2,8-sialyltransferase from mouse lung. J Biochem 118:658-664.

Yoshimi T, Minura N, Aimoto S, Asano A (1993) Transitional expression of neural cell adhesion molecule isoforms during chicken embryonic myogenesis. Cell Struct Funct 18:1-11. 